\title{
PSO-based Resource Allocation in Software-Defined Heterogeneous Cellular Networks
}

\author{
Wenrong Gong ${ }^{1}$, Lihua Pang ${ }^{1}$, Jing Wang ${ }^{1}$ and Meng Xia ${ }^{1}$ \\ ${ }^{1}$ School of Communication and Information Engineering, \\ Xi'an University of Science and Technology, \\ Xi'an, China, 710054 \\ [email: gongwr@163.com] \\ *Corresponding author: Wenrong Gong \\ Received August 31, 2018; revised October 31, 2018; accepted November 27, 2018; \\ published May 31, 2019
}

\begin{abstract}
A heterogeneous cellular network (HCN) is useful to increase the spectral and energy efficiency of wireless networks and to reduce the traffic load from the macro cell. The performance of the secondary user equipment (SUE) is affected by interference from the eNodeB (eNB) in a macro cell. To decrease the interference between the macro cell and the small cell, allocating resources properly is essential to an HCN. This study considers the scenario of a software-defined heterogeneous cellular network and performs the resource allocation process. First, we show the system model of HCN and formulate the optimization problem. The optimization problem is a complex process including power and frequency resource allocation, which imposes an extremely high complexity to the HCN. Therefore, a hierarchical resource allocation scheme is proposed, which including subchannel selection and a particle swarm optimization (PSO)-based power allocation algorithm. Simulation results show that the proposed hierarchical scheme is effective in improving the system capacity and energy efficiency.
\end{abstract}

Keywords: SDN, Heterogeneous Cellular Network, resource allocation, PSO, energy efficiency

This work is supported by the China NSFC Project (Grant No. 61701392/ 61701393), the Science Research Project of Education Department of Shaanxi Province (17JK0514), the Outstanding Youth Science Foundation of Xi'an University Of Science And Technology (2018YQ3-07) 


\section{Introduction}

The rocketing development of smart terminals poses great challenges to data transmission in traditional communication networks and leads to heavy loads at base stations (BSs). The heavy loads of the traditional communication networks make it difficult to transmit data accurately to the terminals with poor channel conditions in a traditional cellular network. Heterogeneous cellular networks (HCNs) are essential for providing a higher throughput in a terminal with poor channel conditions and for increasing the spectral and energy efficiency of wireless networks. Heterogeneous cellular networks comprise a conventional cellular network overlaid with a set of small cells such as picocells or femtocells [1, 2]. However, this network topology will lead to interference between the macro and small cell communication in the hybrid system. To decrease the interference, a proper scheduling mechanism is required to control the transmission power and allocate the system resources.

Coordinate the resources properly is essential for HCNs. There are several kinds of resource allocation ways [3], including co-channel deployment (CCD), orthogonal deployment (OD) and partially shared deployment (PSD). In CCD, all the BSs operate on all available frequencies. In OD, the small-BSs are allocated a part of subcarriers orthogonal to the set of subcarriers been allocated to eNB. In PSD, eNB is allocated some subcarriers for its dedicated use, and some other subcarriers can be shared by eNB and the pico-BSs.

With the study of heterogeneous cellular networks, the complexity of resource allocation algorithms increases, bringing challenges to network response times and data processing capability. A software-defined network (SDN) is a useful technology to address this problem. By decoupling the control plane and the data plane, SDNs enable network administrators to program the network in a dynamic and flexible manner [4-6]. There are some articles that study software-defined heterogeneous cellular networks [7-11]. A hierarchical architecture for an SDN-based heterogeneous cellular network and the management of software-defined heterogeneous cellular networks were studied in [7] and [8]. Reference [9] proposed an SDN system model to assist in frequency resource allocation and interference elimination. However, the studies about software defined heterogeneous networks are not yet fully engaged. To make the study of software-defined heterogeneous networks more systematic, there is still much work to be done.

Based on the above analysis, in this paper, we study the resource allocation of software-defined network-based heterogeneous cellular networks (SDHCNs) and propose a particle swarm optimization (PSO)-based power allocation scheme. First, we build the SDHCNs system model and formulate the optimization problem. Second, a greedy subcarrier selection method is proposed. Then, to guarantee the interference received by user equipment (UE) does not affect the data rate performance and obtain an energy efficiency promotion, we propose a power allocation algorithm based on PSO. Simulation results show that the proposed scheme improves the energy efficiency and system capability.

The remainder of this paper is organized as follows. In Section 2, we discuss related works, and the system scenarios and the problem formulation are introduced in Section 3. In Section 4, the specific steps of the proposed resource allocation scheme are given. In Section 5, we evaluate the performance of proposed scheme and present the simulation results and analysis, which demonstrating the improved performance of our proposed scheme in comparison to systems with other resource allocation schemes. Finally, the conclusion of this paper is shown in Section 6. 


\section{Related Work}

Allocating resources properly is essential to keep interference within a reasonable range for UE in a heterogeneous cellular network. Much literature has studied the resource allocation of heterogeneous cellular networks. The optimization problems for the resource allocation of heterogeneous cellular networks can be roughly classified into the following cases.

1) Maximizing the system capacity of a heterogeneous cellular network and guaranteeing the data rate request of UEs [12, 13]. In [12], to maximize the total system profit, a user association and downlink resource allocation scheme was proposed. In [13], a joint subchannel and power allocation scheme was proposed in indoor dense environments to improve the system capacity.

2) Maximizing the data rate of a secondary UE (SUE) while guaranteeing the QoS of the primary UE (PUE) [14]. To maximize the data rate of SUEs, an efficient frequency allocation scheme was proposed in [14].

3) Minimizing the interference between eNBs and low-power BSs [15-17]. To reduce the interference between eNBs and low-power BSs within the coverage area, a distributed resource allocation algorithm was proposed in [15]. In [16], a fast frequency resource allocation scheme was proposed to mitigate interference in heterogeneous networks by graph theory. To mitigate serious intercell interference between the dense small cells, an enhanced small cell grouping based intercell interference control scheme was proposed in [17].

4) Maximizing the energy efficiency of the system [18-21]. Energy efficiency has become an increasingly important issue in wireless communication because of the increasing energy cost and concern over environmental issues. The resource optimization problem was formulated as a nonconcave energy-efficient maximization problem, and an energy-efficient allocation algorithm which joint power and frequency resource was developed in [18]. To improve the system energy efficiency and satisfying the rate requirement of UEs, a resource allocation algorithm was proposed in [19], which included the radius selection, resource scheduling, and power allocation. An optimal partial spectrum reuse (PSR) scheme was derived in [20], and the spectrum share scheme in HCNs was analyzed.

These studies improve the system performance to some extent. However, the growing complexity of the studies about HCNs brings challenges to the core network. As a result of adopting a more complex distributed control, the resource allocation of HCNs will produce a larger signaling load.

An SDN is a promising technology to settle these problems. There are several advantages of a software defined HCNs. First, with a centralized SDN controller, the energy efficiency of a software defined HCN's architecture can be improved by reducing the number of communication links. Second, the global network view of SDN controller makes the management and optimization of resource allocation much more efficiently [6]. Therefore, we combine an SDN with a heterogeneous cellular network and propose an SDN-based wireless network architecture in this article. The proposed SDN-based wireless network architecture can also be applied in other wireless network scenarios to offload the burden on the network, such as a wireless sensor network [22], a satellite communication network [23] and the internet of thing (IoT) system [24]. Since energy efficiency has become an increasingly important issue in wireless communication, the optimization problem in this paper is modeled to maximize the system energy efficiency. 


\section{System Model and Problem Formulation}

We show the system model of software defined HCN in this section and illustrate the problem of resource allocation.

\subsection{System Model}

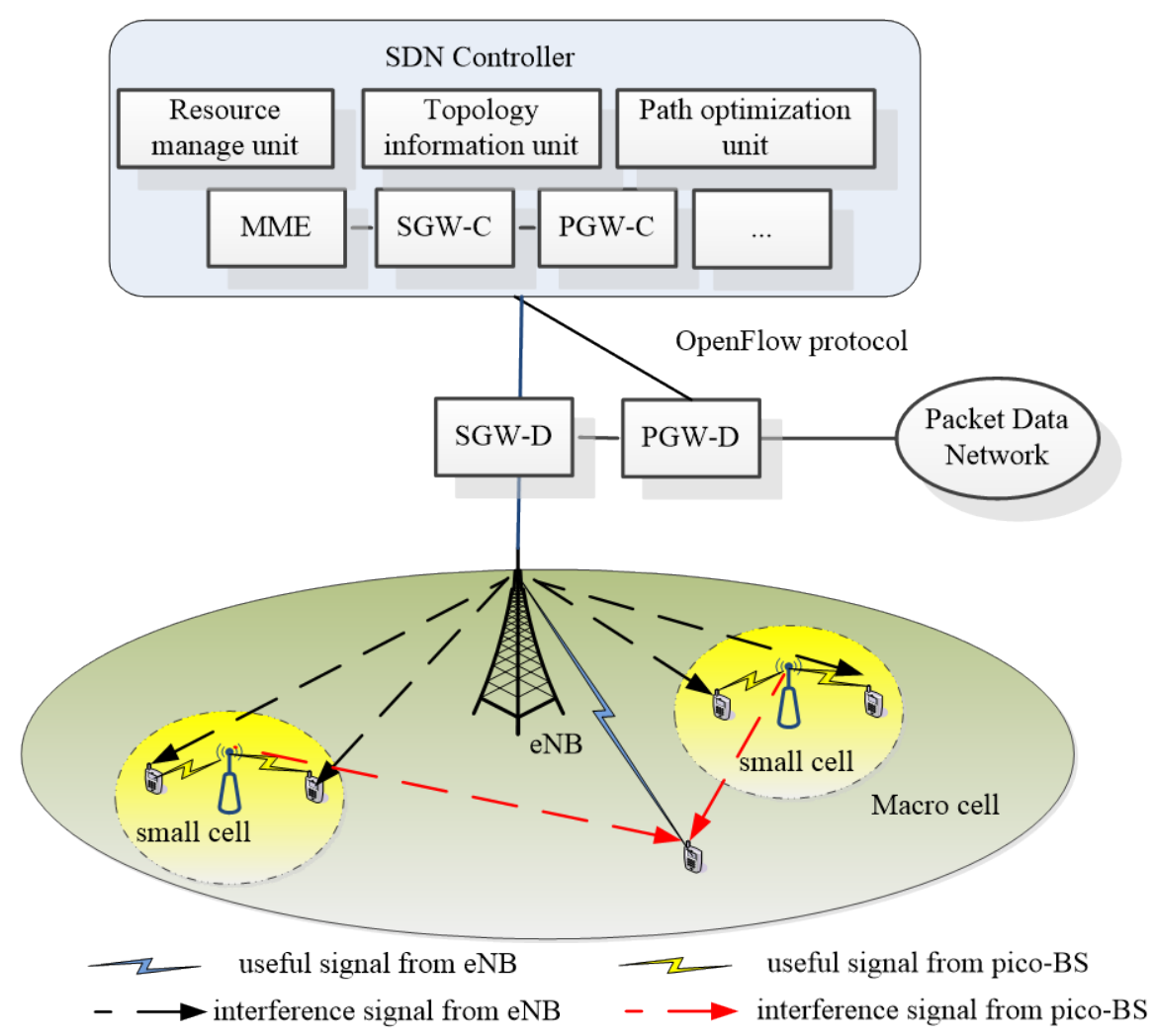

Fig. 1. System model

In this paper, we consider a SDHCN where a primary network and $M$ small cells coexist in the heterogeneous cellular network system, as shown in Fig. 1. In this network, there is an SDN controller with a mobility management entity (MME), the SGW control unit (SGW-C), and the PGW control unit (PGW-C). The data plane includes the SGW data unit (SGW-D) and the PGW data unit (PGW-D). The control plane and data plane communicate with each other via the OpenFlow protocol. It is assumed that $I$ PUEs are located in the SDHCN and $N$ SUEs are located in each small cell. We assume that the location of the UEs obeys independent homogeneous Poisson point process (HPPP). UEs' location information can be obtained from the SDN controller. Each subchannel is a physical resource block (PRB) with a bandwidth of $B$. We assume that the frequency used by the macro base station can be used by the small cell. The downlink resource sharing is considered in this article with Rayleigh fading channels. In the downlink, the frequency resources allocated to each PUE are mutually orthogonal at the same time. 


\subsection{Problem Formulation}

For the $i_{\text {th }}$ PUE in the macro cell, the data rate can be written as

$$
r_{i}=\sum_{k=1}^{K} B \log _{2}\left(1+\frac{a_{i}^{(k)} p_{e N B}^{(k)} h_{e N B 2 i}^{(k)}}{I_{i}^{(k)}+B P_{N_{0}}}\right),
$$

where $B$ is the bandwidth of each subchannel, $K$ is the number of subchannels available for PUEs, $a_{i}^{(k)}$ is an allocation indicator variable, when the $k_{t h}$ subchannel is allocated to PUE $i$, $a_{i}^{(k)}=1$, otherwise, $a_{i}^{(k)}=0 . p_{e N B}^{(k)}$ is the eNB transmission power on the $k_{t h}$ subchannel, $h_{e N B 2 i}^{(k)}$ denotes the channel condition between the eNB and the $i_{t h}$ PUE on subchannel $k, I_{i}^{(k)}$ denotes interferences received by the $i_{\text {th }}$ PUE on subchannel $k$, and $P_{N_{0}}$ is the noise power per subchannel.

The total energy efficiency of the macro cell can be calculated as

$$
E E_{\text {macro }}=\frac{\sum_{i=1}^{I} r_{i}^{(k)}}{\sum_{i=1}^{I} \sum_{k=1}^{K} p_{\text {eNB2i }}^{(k)}} .
$$

For the $n_{\text {th }}$ SUE in the $m_{t h}$ small cell, the data rate can be written as

$$
r_{m, n}=\sum_{k=1}^{K} B \log _{2}\left(1+\frac{b_{m, n}^{(k)} p_{m, n}^{(k)} h_{m, n}^{(k)}}{I_{m, n}^{(k)}+B P_{N_{0}}}\right),
$$

where $B$ is the bandwidth of each subchannel, $b_{m, n}^{(k)}$ is also an allocation indicator variable, namely, $b_{m, n}^{(k)}=1$ indicates that subchannel $k$ is allocated to SUE $n$ in the $m_{\text {th }}$ small cell; otherwise, $b_{m, n}^{(k)}=0 . p_{m, n}^{(k)}$ is the transmit power of the pico-BS in the $m_{t h}$ small cell on subchannel $k, h_{m, n}^{(k)}$ denotes the channel condition between the $m_{\text {th }}$ pico-BS and the $n_{t h}$ user equipment on subchannel $k, I_{m, n}^{(k)}$ denotes interferences received by the $n_{t h}$ user equipment in the $m_{t h}$ small cell on subchannel $k$, and $P_{N_{0}}$ is the noise power per subchannel.

The small cell energy efficiency is given by

$$
E E_{\text {small }}=\frac{\sum_{m=1}^{M} \sum_{n=1}^{N} r_{m, n}}{\sum_{m=1}^{M} \sum_{n=1}^{N} \sum_{k=1}^{K} p_{m, n}^{(k)}} .
$$

We can obtain the total energy efficiency of heterogeneous cellular network:

$$
E E=\frac{\sum_{i=1}^{I} r_{i}^{(k)}+\sum_{m=1}^{M} \sum_{n=1}^{N} r_{m, n}}{\sum_{i=1}^{I} \sum_{k=1}^{K} p_{e N B 2 i}^{(k)}+\sum_{m=1}^{M} \sum_{n=1}^{N} \sum_{k=1}^{K} p_{m, n}^{(k)}} .
$$

The optimization problem is modeled by maximizing the energy efficiency of the small cell, which is given by 


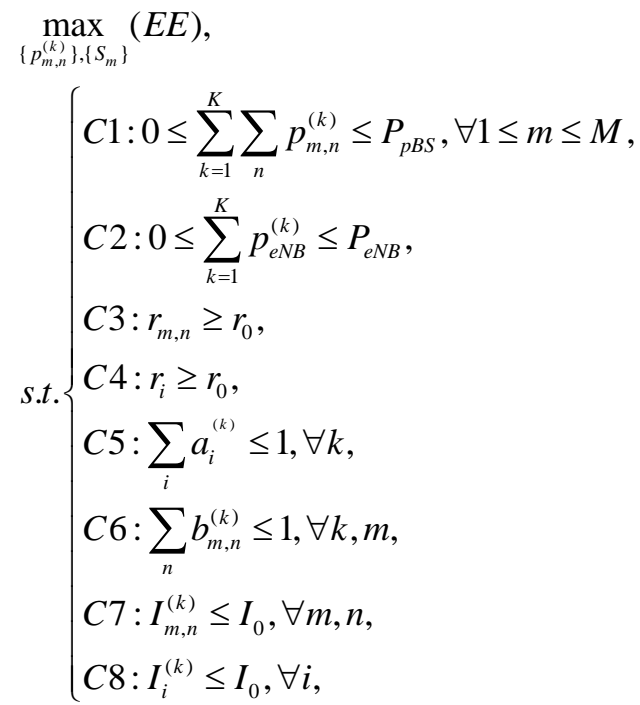

where $P_{p B S}$ and $P_{e N B}$ are the maximum available power of each pico-BS and eNB, respectively, $r_{0}$ is the data rate required by the $\mathrm{UE}$, and $I_{0}$ is the maximum value of interference that one UE can withstand. C1 guarantees that the sum of the power allocated to each subchannel of one pico-BS is not greater than the maximum available power of each pico-BS. C2 guarantees that the sum of the power allocated to each subchannel of the eNB is not greater than the maximum available power of the eNB. C3 ensures that the data rate requirement of each UE in small cell is satisfied. C4 represents that the required data rate of UE in macro cell should be satisfied. C5 and C6 represent that each subchannel can be allocated to one potential link at most in macro cell or in one small cell. C7 and C8 guarantee that the interference received by one UE should be smaller than a threshold value.

Since it would be unrealistic to get all of the channel conditions and the optimization problem in (6) includes both discrete and continuous variables, the optimal solution to (6) is not feasible. In light of these restrictions, we propose a hierarchical scheme to achieve a suboptimal solution.

\section{Hierarchical Resource Allocation Scheme}

\subsection{Subchannel selection}

We assume that the UE in a small cell can reuse downlink resources of the macro cell. Different small cells could transmit a signal at the same time. Therefore, the interference received by the $n_{t h}$ UE in the $m_{t h}$ small cell on subchannel $k$ can be calculated by

$$
I_{m, n}^{(k)}=P_{e N B}^{(k)} h_{e N B 2 m n}^{(k)}{ }^{2} d_{e N B 2 m n}^{-\alpha}+\sum_{m^{\prime}=1, m^{\prime} \neq m}^{M} P_{p B S_{m^{\prime}}}^{(k)} h_{m^{\prime} 2 m n}^{(k)}{ }^{2} d_{m^{\prime} 2 m n}^{-\alpha},
$$

where $P_{e N B}^{(k)}$ and $P_{p B S_{m^{\prime}}}^{(k)}$ denote the transmission power of the eNB and the $m_{t h}{ }^{\prime}$ pico-BS on subchannel $k$, respectively, $h_{e N B 2 m n}^{(k)}$ and $d_{e N B 2 m n}$ denote the channel condition and the distance between the eNB and receiver $n$ in the $m_{t h}$ small cell on subchannel $k$, and $h_{m^{\prime} 2 m n}^{(k)}$ $d_{m^{\prime} 2 m n}$ denote the channel condition and distance between the $m_{t h}{ }^{\prime}$ pico-BS and receiver $n$ in 
the $m_{t h}$ small cell on subchannel $k$.

The interference experienced by the $i_{\text {th }}$ PUE that occupied the subchannel $k$ is given by

$$
I_{i}^{(k)}=\sum_{m=1}^{M} P_{p B S_{m}}^{(k)} h_{p B S_{m} 2 i}^{(k)}{ }^{2} d_{p B S_{m} 2 i}^{-\alpha},
$$

where $P_{p B S_{m}}^{(k)}$ is the transmission power of the $m_{t h}$ pico-BS on subchannel $k, h_{p B S m 2 i}^{(k)}$ denotes the channel condition between the $m_{t h}$ pico-BS and receiver $i_{t h}$ PUE on subchannel $k$, and $d_{p B S_{m} 2 i}$ denotes the distance between the $m_{t h}$ pico-BS and receiver $i_{t h}$ PUE.

To mitigate interference between eNB and pico-BSs, the subchannel selection optimization problem is given by

$$
\left\{k_{1,1}, \cdots, k_{m, n}, \cdots\right\}=\underset{k=1, \cdots, K}{\arg }\left(\min \left(\sum_{n=1}^{N} \sum_{m=1}^{M} I_{m, n}^{(k)}+\sum_{i=1}^{I} I_{i}^{(k)}\right)\right) .
$$

A greedy PRB selection algorithm (GPSA) is proposed to settle the problem in (9). Table 1 specifically shows the proposed GPSA.

Table 1. Greedy PRB Selection Algorithm (GPSA)

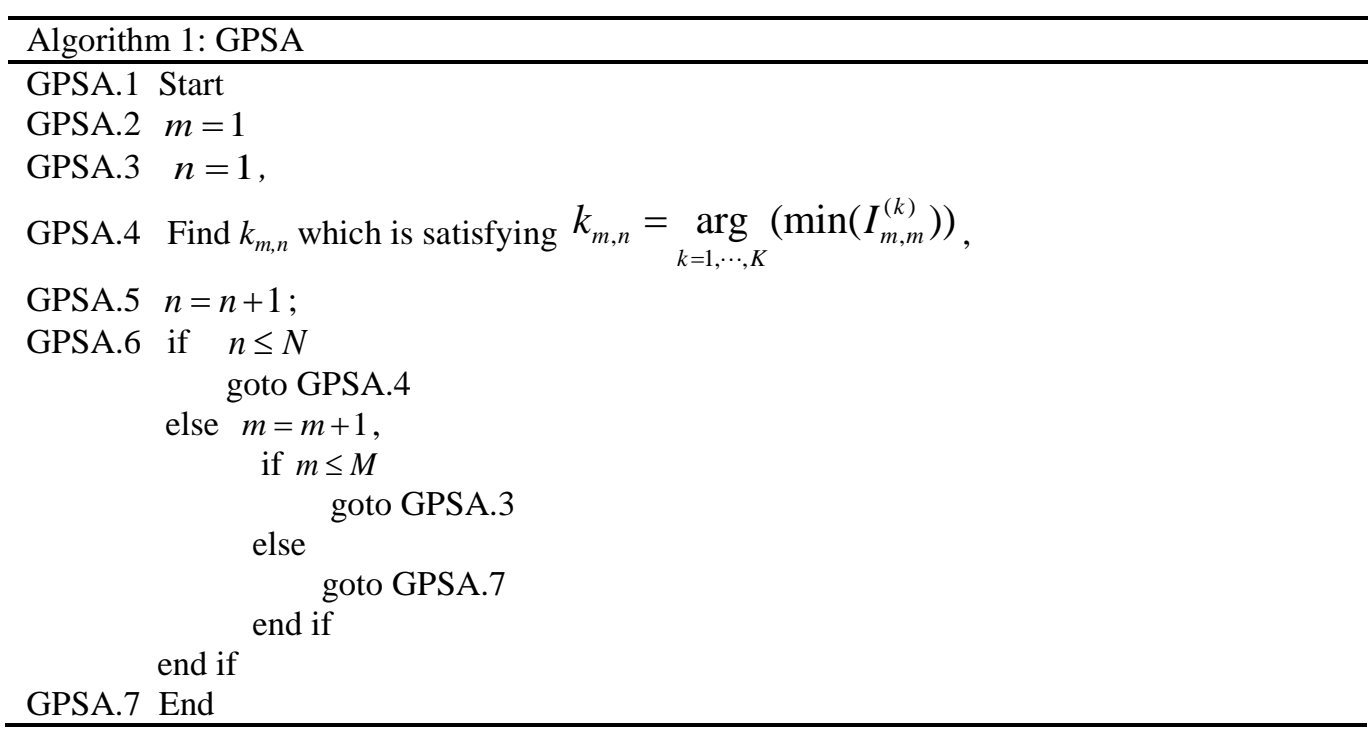

In the greedy PRB selection algorithm, each UE in the small cell finds the subchannel with the lowest received interference. The complexity of this procedure is of the order $O(M N)$.

\subsection{PSO-based power allocation Algorithm}

After selectting subchannels, a particle swarm optimization-based power allocation scheme is proposed to maximize the energy efficiency (PPA-MEE). The PPA-MEE algorithm is summarized in Table 2. 
Table 2. PPA-MEE Algorithm

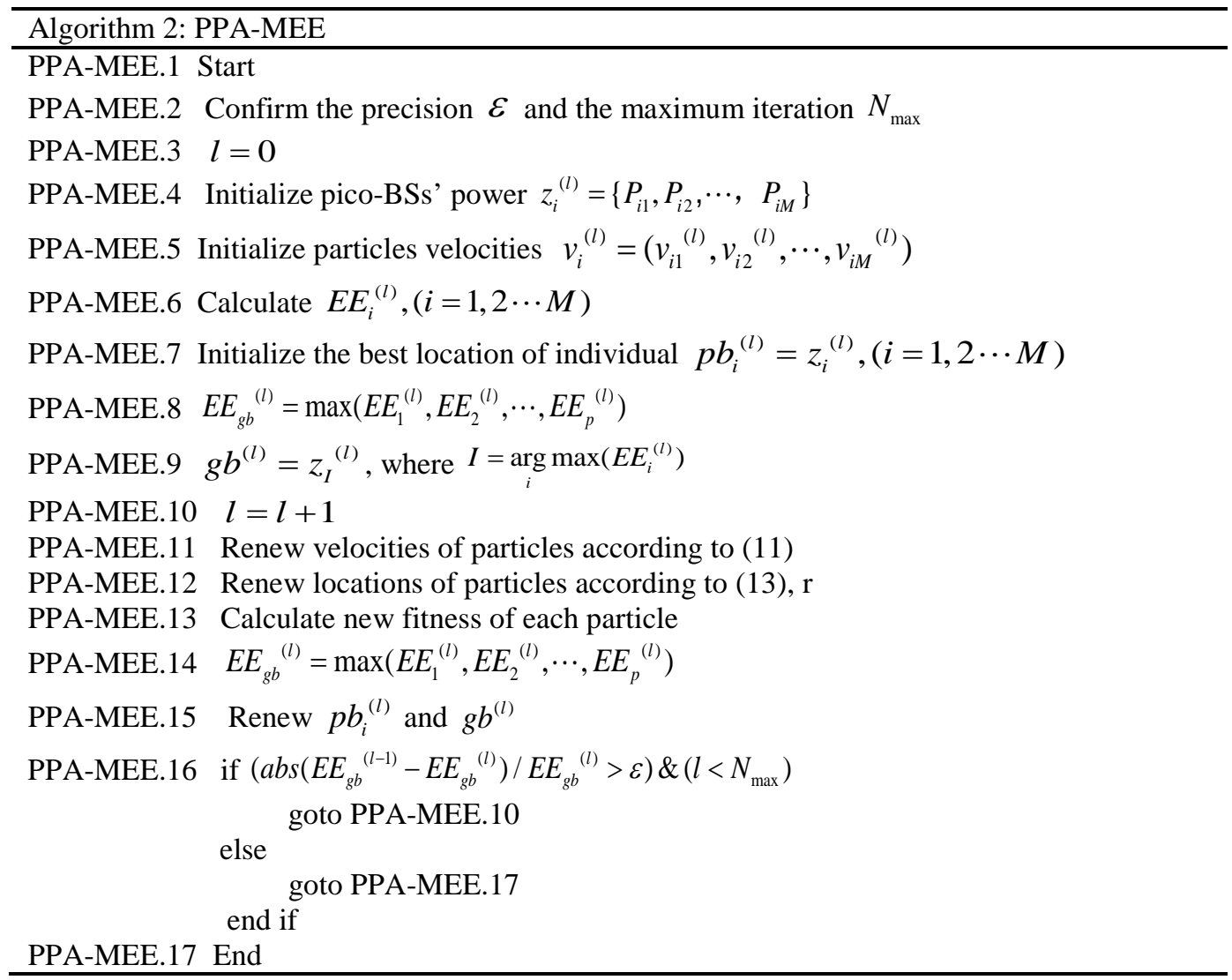

Firstly, we need to confirm the precision $\varepsilon$ and the maximum iteration $N_{\max }$. The location of the $i_{\text {th }}$ particle $z_{i}^{(l)}=\left\{P_{i 1}, P_{i 2}, \cdots, P_{i M}\right\}$ is set as the power allocated to the pico-BSs.

$$
z_{i}=\left\{P_{i 1}, P_{i 2}, \cdots, P_{i M}\right\}
$$

Since the dimension of the particles in the PSO algorithm is $M$, the PSO algorithm of power allocation is $M$-dimensional. We denote $p$ as the number of particles.

Then, we calculate the fitness of each particle $E E_{i}$, and make $E E_{i}$ as the initial best location of the individual particle. We choose the maximum value of each initial positions as the first global best location.

The velocities of the particles are calculated according to formula (11),

$$
\begin{aligned}
& v_{i m}{ }^{(l+1)}=\omega^{(l)} v_{i m}{ }^{(l)}+c_{1} r_{1}\left(p b_{i}^{(l)}-z_{i}^{(l)}\right)+c_{2} r_{2}\left(g b^{(l)}-z_{i}^{(l)}\right), \\
& v_{i}^{(l)}=\left(v_{i 1}{ }^{(l)}, v_{i 2}{ }^{(l)}, \cdots v_{i M}{ }^{(l)}\right),
\end{aligned}
$$

where $\omega$ is the inertial weight and value between 0 and 1 .

$\omega$ in the $l_{t h}$ iteration is given by

$$
\omega^{(l)}=\frac{\omega_{\text {start }}-\left(\omega_{\text {start }}-\omega_{\text {end }}\right) l^{2}}{N_{\max }^{2}},
$$

The value of $\omega_{\text {start }}$ is 0.9 and the value of $\omega_{\text {end }}$ is 0.4 in this paper.

The location of the $i_{t h}$ particle is computed as: 


$$
Z_{i}^{(l+1)}=Z_{i}^{(l)}+v_{i}^{(l+1)} .
$$

The new fitness of each particle is calculated and the privately optimal and global optimum fitness are renewed. After that, the process is repeated starting at PPA-MEE.10 until the maximum iteration is reached or the difference between two fitness drops below the minimum threshold level $\varepsilon$.

To obtain the best location of a particle, the power allocation algorithm consists of two parts. The initialization procedure (PPA-MEE. 1 -PPA-MEE. 9) has a complexity of $O(M)$, where $M$ denotes the number of small cells in HCNs. The complexity of the iteration procedure (DRAA. 10 -DRAA. 17) is $O\left(N_{\max } M\right)$ in the worst case, where $N_{\max }$ is the maximum iteration number. Therefore, the complexity of PPA-MEE is $O\left(N_{\max } M\right)$.

\section{Simulation Results}

To evaluate the performance of the proposed algorithm, simulation in MATLAB is performed. We consider a heterogeneous cellular network system where a primary network and a secondary network coexist. UE is randomly distributed in the cell. The proposed scheme is compared with other two schemes. The first one is the water-filling power allocation (WFPA) scheme, and the second one is the average power allocation (APA) method. Our simulation parameters are listed in Table 3.

Table 3. SIMULATION PARAMETERS

\begin{tabular}{ll}
\hline PARAMETER & VALUE \\
\hline Micro cell redius & $500 \mathrm{~m}$ \\
Small cell radius & $10 \mathrm{~m}$ \\
The number of UEs in each small cell & 20 (Except for figure 2 and figure 3) \\
The number of UEs in macro cell & 80 (Except for figure 2 and figure 3) \\
SINR $_{0}$ & $10 \mathrm{~dB}$ (Except for figure 4 and figure 5) \\
The transmission power of eNB & $36 \mathrm{dBm}$ \\
The transmission power of Small cell BS & $20 \mathrm{dBm}$ \\
Noise spectral density & $-174 \mathrm{dBm} / \mathrm{Hz}$ \\
The bandwidth of each PRB & $180 \mathrm{kHz}$ \\
Available bandwidth & $10 \mathrm{MHz}$ \\
The number of particles in PSO algorithm $(p)$ & 40 \\
$\omega_{\text {start }} / \omega_{\text {end }}$ & $0.9 / 0.4$ \\
$c 1, c 2$ & 1.4962 \\
$N_{\text {max }}$ & 1500 \\
$\varepsilon$ & $10^{-6}$ \\
\hline
\end{tabular}




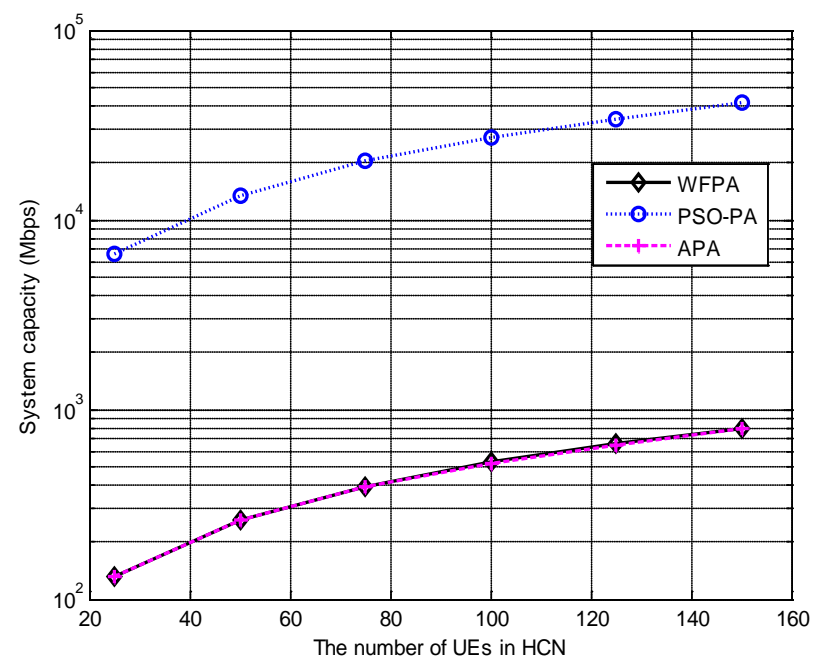

Fig. 2. System capacity vs. the amount of UE in the HCNs

Fig. 2 illustrates the system capacity performance with the different methods against the amount of UE in the HCNs. It reveals that by applying the proposed scheme, the system capacity is improved. For example, when the amount of UE in the HCNs is equal to 100, the system capacity with the proposed scheme is approximately $2 * 10^{4} \mathrm{Mbps}$, while the system capacity of the system with the WFPA scheme and the APA scheme are approximately $4 * 10^{2}$ Mbps. As the amount of UE increases, the system capacity of the three schemes increases. Our proposed scheme can obtain the highest system capacity because it adjusts the power and the number of PRBs allocated to each UE adaptively, according to different data rate demands of the UE. In addition, the system capacity of the system with the WFPA scheme and the APA scheme is nearly always the same. This occurs because the water-filling power allocation scheme performs like the APA method when the channel is well conditioned.

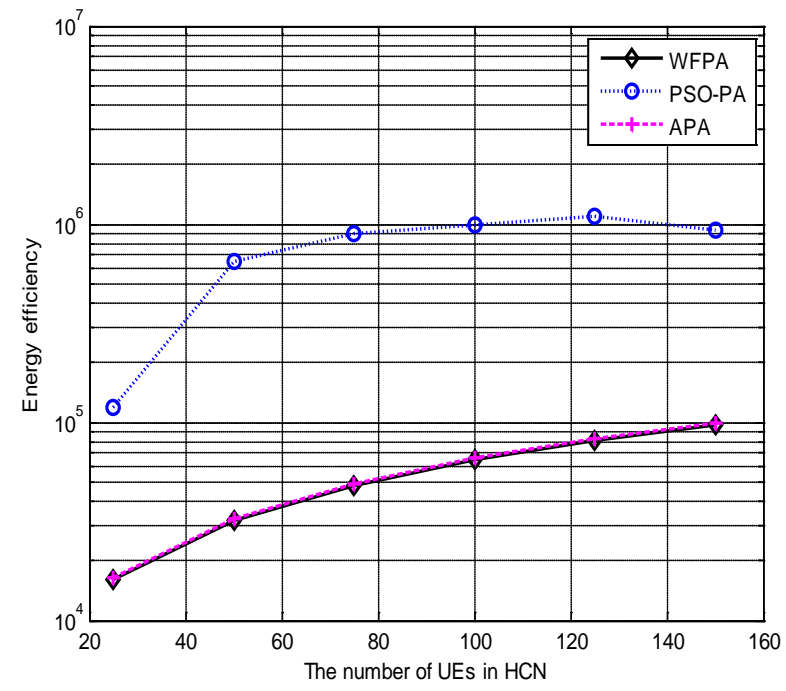

Fig. 3. Energy efficiency vs. the amount of UE in the HCNs 
Fig. 3 shows the energy efficiency of the system with different resource allocation schemes versus the amount of UE in the HCNs. From this figure, we can make several observations. First, the energy efficiency of the system with the proposed resource allocation scheme is always the highest. The spectral efficiencies of system with the WFPA scheme and the APA scheme are much lower. The highest energy efficiency of our proposed method is attributed to the subchannels selection and the PSO-based power allocation. Second, the energy efficiency of all these methods increases with an increasing amount of UEs. The greater the amount of UEs a system has, the more system capability they obtain. However, the total consumed power remains unchanged, and thus, the energy efficiency improved. In addition, the energy efficiencies of the system with the WFPA scheme and that with the APA scheme are almost the same because the WFPA scheme performs like the APA scheme when the channel is well conditioned.

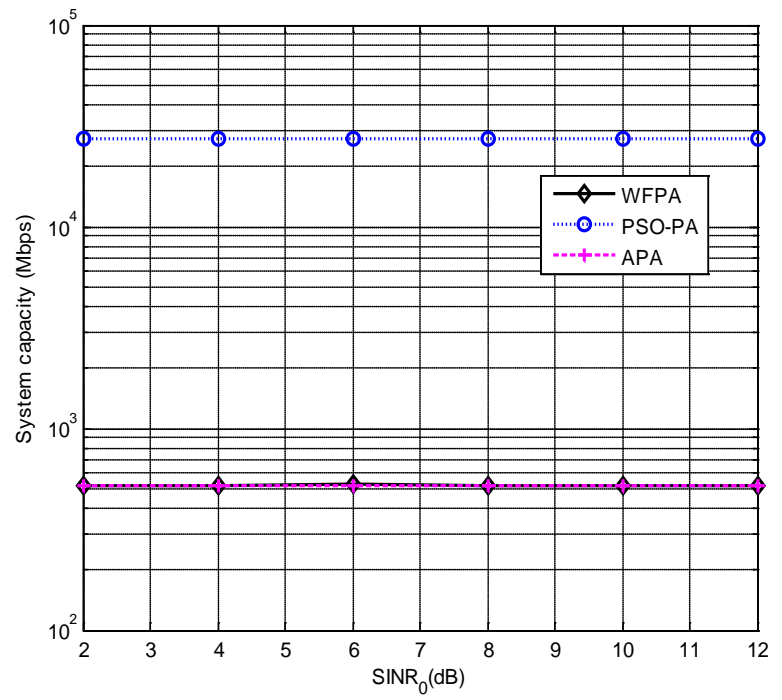

Fig. 4. System capacity vs. $\mathrm{SINR}_{0}$

The system capacity performance with the different methods versus the threshold signal-to-interference plus noise ratio (SINR) of the UE is shown in Fig. 4. We can see that with the same $\mathrm{SINR}_{0}$ of the UE, the system capacity with the proposed method is the highest. This illustrates that our proposed method can improve the system capacity. Second, the system capacities with the WFPA and APA methods perform the same. In addition, the system capabilities with three methods basically have no variation as the $\mathrm{SINR}_{0}$ increases. The SINR performances of UEs are higher than the setting threshold SINR, so the threshold SINR cannot affect the performance of the system capacity. This illustrates that the system with the proposed method can maintain stable high capacity performance. 


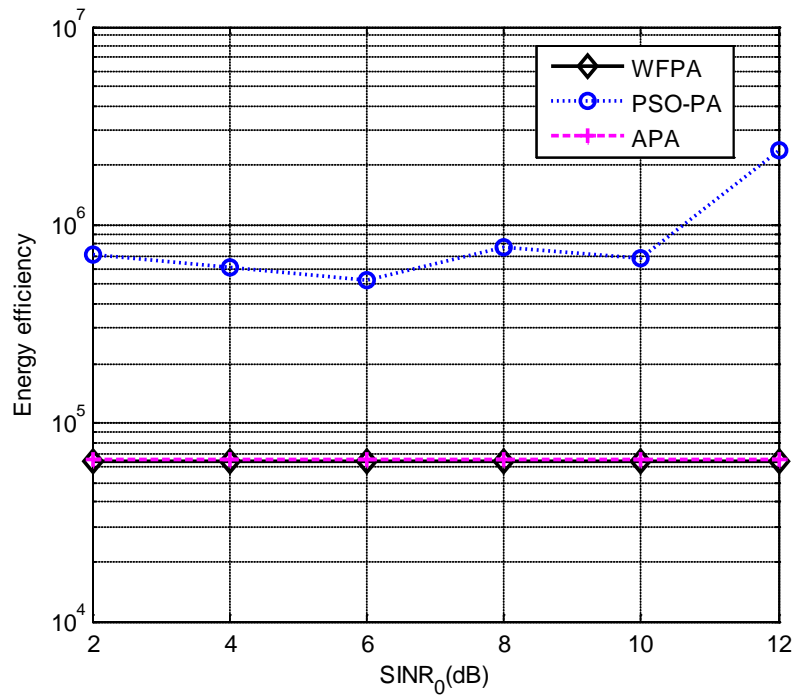

Fig. 5. Energy efficiency v.s. SINR $_{0}$

Fig. 5 illustrates the energy efficiency of a system with different resource allocation schemes against the SINR threshold of the UE. From this figure, we have the following observations. First, the energy efficiency of the system with the proposed resource allocation scheme is always the highest. Second, the system capability with the proposed scheme increases as the $\mathrm{SINR}_{0}$ increases, while the system capabilities with the other two methods basically have no variation as the $\operatorname{SINR}_{0}$ increases. This illustrates the much lower power consumption of the proposed scheme when the SINR threshold of the UE increases. Additionally, it can be concluded that a high energy efficiency performance can be achieved by the system stably with the proposed scheme.

\section{Conclusions}

A hierarchical resource allocation scheme of a software-defined heterogeneous cellular network is proposed in this article, which includes subchannel selection and power allocation. First, a software-defined network system model is built to assist the system in carrying out the resource allocation procedure. With the constraint to minimize the sum interference of the system, a greedy PRB selection algorithm is proposed. Then, we proposed a PSO-based power allocation scheme to optimize the system energy efficiency. The proposed scheme ensures that the system allocates the appropriate subchannels to the UE successfully, and each subchannel has been allocated suitable power. The simulation results demonstrate that the energy efficiency and system capability of the system improved greatly by our proposed resource allocation scheme.

\section{References}

[1] Ismail, Muhammad, Atef Abdrabou, and Weihua Zhuang. "Cooperative Decentralized Resource Allocation in Heterogeneous Wireless Access Medium,” IEEE Transactions on wireless communications, vol. 12, no. 2, pp. 714-724, February, 2013. Article (CrossRef Link). 
[2] Yantian Hou, Ming Li, Xu Yuan, Y. Thomas Hou and Wenjing Lou, "Cooperative Interference Mitigation for Heterogeneous Multi-hop Wireless Networks Coexistence,” IEEE Transactions on Wireless Communications, vol. 15, no. 8, pp. 5328 - 5340, August, 2016. Article (CrossRef Link).

[3] Ghimire, Jagadish and Catherine Rosenberg. "Resource allocation, transmission coordination and user association in heterogeneous networks: A flow-based unified approach,” IEEE Transactions on Wireless Communications, vol. 12, no.3, pp. 1340-1351, March, 2013. Article (CrossRef Link).

[4] A. Jain, N. S. Sadagopan, S. K. Lohani and M. Vutukuru, "A comparison of sdn and nfv for re-designing the lte packet core,” in IEEE Conference on Network Function Virtualization and Software Defined Networks (NFVSDN), pp. 74-80, November 7-10, 2016. Article (CrossRef Link).

[5] Y. Zhu, F. Yan, Y. Zhang, R. Zhang and L. Shen, "SDN-Based Anchor Scheduling Scheme for Localization in Heterogeneous WSNs," IEEE Communications Letters, vol. 21, no. 5, pp. 1127-1130, May, 2017. Article (CrossRef Link).

[6] N. A. Jagadeesan and B. Krishnamachari, "Software-Defined Networking Paradigms in Wireless Networks: A Survey,” Acm Computing Surveys, vol. 47, no. 2, pp.1-11, January 2015.

Article (CrossRef Link).

[7] Shu Y. "Heterogeneous Networking Architecture Based on SDN," Chinese Journal of Electronics, vol. 26, no. 1, pp. 166-171, January 2017. Article (CrossRef Link).

[8] Nayak, Akshatha M., et al. "Control and Management of Heterogeneous RATs in 5G Wireless Networks: An SDN/NFV Approach ,” 2018.

[9] Kakalou I, Psannis K, Siakavara K, Goudos S K. and Ishibashi Y, "SDN-based QoS Provisioning and Interference Management in Heterogeneous CRN," IEEE International Conference on Modern Circuits and Systems Technologies, pp. 1-4, 7-9 May, 2018. Article (CrossRef Link).

[10] Sun S, Gong L, Rong B and Lu K. "An intelligent SDN framework for 5G heterogeneous networks,” IEEE Communications Magazine, vol. 53, no. 11, pp. 142-147, November 2015. Article (CrossRef Link).

[11] Robinson M, Milosavljevic M, Kourtessis P, Fisher S, Stafford G P. and Treiber J. “QoE based holistic traffic engineering in SDN enabled heterogeneous transport networks," International Conference on Transparent Optical Networks. pp. 1-4, July 2-6, 2017. Article (CrossRef Link).

[12] Zalghout M, Helard J, Crussiere M, Abdul-Nabi S and Khalil A, “A Greedy Heuristic Algorithm for Context-Aware User Association and Resource Allocation in Heterogeneous Wireless Networks," in IEEE 86th Vehicular Technology Conference (VTC-Fall), September 24-27, 2017. Article (CrossRef Link).

[13] Kim, J., and Cho, D. H., “A Joint Power and Subchannel Allocation Scheme Maximizing System Capacity in Indoor Dense Mobile Communication Systems,” IEEE Transactions on Vehicular Technology, vol. 59, no. 9, pp. 4340 - 4353, November, 2010. Article (CrossRef Link).

[14] Wei Bao, and Ben Liang. "Rate Maximization through Structured Spectrum Allocation and User Association in Heterogeneous Cellular Networks,” IEEE Transactions on Communications, vol. 63, no. 11, pp. 4510-4524, November, 2015. Article (CrossRef Link).

[15] Song Q, Wang X, Qiu T and Ning Z, “An Interference Coordination-Based Distributed Resource Allocation Scheme in Heterogeneous Cellular Networks,” IEEE Access, 5, pp.2152-2162, January, 2017. Article (CrossRef Link).

[16] Niu C, Li Y, Hu R Q and Ye F, "Fast and Efficient Radio Resource Allocation in Dynamic Ultra-Dense Heterogeneous Networks,” IEEE Access , 5, pp.1911-1924, February 2017. Article (CrossRef Link).

[17] Huang J, Zhang $\mathrm{H}, \mathrm{Xu} \mathrm{W}$ and Zhang $\mathrm{H}$, "Grouping based inter-cell interference coordination in LTE-A dense small-cell networks,” IEEE International Symposium on Microwave, Antenna, Propagation and EMC Technologies for Wireless Communications (MAPE), pp.78 - 83, October, 2013. Article (CrossRef Link).

[18] Runzi Liu, Min Sheng, and Weihua Wu. "Energy-Efficient Resource Allocation for Heterogeneous Wireless Network with Multi-homed User Equipments,” IEEE Access , vol. 6, pp. 14591- 14601, February 2018. Article (CrossRef Link). 
[19] Coskun, Cemil Can, Kemal Davaslioglu, and Ender Ayanoglu. "Three-Stage Resource Allocation Algorithm for Energy-Efficient Heterogeneous Networks,” IEEE Transactions on Vehicular Technology, vol.66. no.8, pp.6942-6957. August 2017. Article (CrossRef Link).

[20] Cao, Dongxu, Sheng Zhou, and Zhisheng Niu. "Improving the Energy Efficiency of Two-Tier Heterogeneous Cellular Networks through Partial Spectrum Reuse,” IEEE Transactions on Wireless Communications, vol.12, no.8, pp. 4129-4141, August 2013. Article (CrossRef Link).

[21] Eryigit, Salim, Suzan Bayhan, and Tuna Tugcu. "Energy-Efficient Multichannel Cooperative Sensing Scheduling With Heterogeneous Channel Conditions for Cognitive Radio Networks,” IEEE transactions on vehicular technology, vol.62. no.6 , pp. 2690-2699. July 2013. Article (CrossRef Link).

[22] Jingtao Su, Shengsong Yang, Haitao Xu and Xianwei Zhou, “A Stackelberg Differential Game based Bandwidth Allocation in Satellite Communication Network," China Communications, vol.15, no.8, pp. 205-214, August 2018. Article (CrossRef Link).

[23] Haitao Xu, Chao Guo and Long Zhang. "Optimal Power Control in Wireless Powered Sensor Networks: A Dynamic Game Based Approach,” Sensors, vol.17, no.3, pp. 547, March 2017. Article (CrossRef Link).

[24] Jingtao Su, Haitao Xu, Ning Xin, Guixing Cao and Xianwei Zhou, "Resource Allocation in Wireless Powered IoT System: a Mean Field Stackelberg Game based Approach,” Sensors, vol.18, no.10, pp.3173, September 2018. Article (CrossRef Link). 


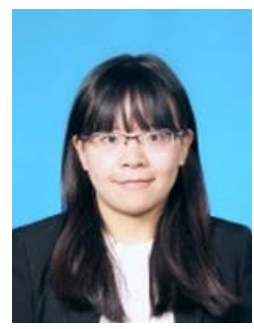

Wenrong Gong was born in 1989. She received the Ph.D. degree in 2015 from Beijing University of Posts and Telecommunications, Beijing. She is currently a Lecturer with the School of Communication and Information Engineering, Xi'an University of Science and Technology, Xi'an. Her current research interests include D2D communication, software defined network, multimedia broadcast/multicast service systems and radio resource management in wireless communication networks.

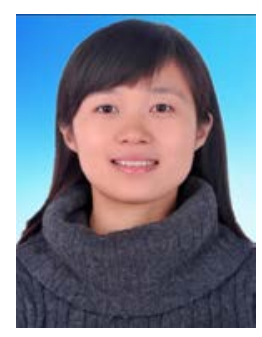

Lihua Pang received the B.E., M.S., and Ph.D. degrees from Xidian University, Xi'an, China, in 2006, 2009, and 2013, respectively, all in electrical engineering. She is currently an Associate Professor with the School of Information Engineering, Xi'an University of Science and Technology, Xi'an. Her current research interests are signal processing for wireless communications, stochastic network optimization, and network performance analysis.

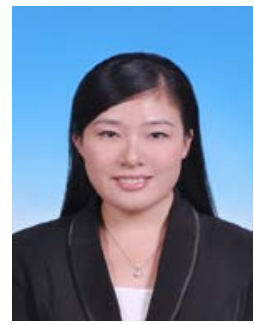

Jing Wang received the Ph.D. degree from the Institute of Electronics, Chinese Academy of Sciences, Beijing, China, in 2012. She is currently a Lecturer with the School of Communication and Information Engineering, Xi'an University of Science and Technology, Xi'an. Her current research interests include software-defined radios and real signal processing for interferometric synthetic-aperture-radar systems.

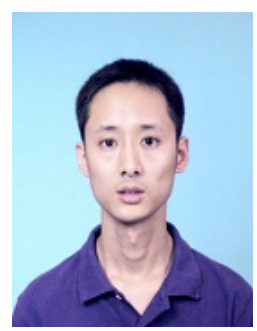

Meng Xia, was born in Ankang, Shaanxi Province, China, in January 1981. He received his M.S. and Ph.D. degrees from Xidian University, Xi'an, China, in March 2007 and December 2012, respectively. He is currently a Lecturer with the School of Communication and Information Engineering, Xi'an University of Science and Technology, Xi'an. His research interests include software radio, signal processing, ground moving target indication etc. 